\title{
ARTICLE
}

Clinical Study

\section{Early metabolic response in sequential FDG-PET/CT under cetuximab is a predictive marker for clinical response in first- line metastatic colorectal cancer patients: results of the phase II REMOTUX trial}

\author{
Anne Katrin Berger ${ }^{1}$, Stephan Lücke ${ }^{2}$, Ulrich Abel ${ }^{2}$, Georg Martin Haag ${ }^{1}$, Carsten Grüllich ${ }^{1}$, Annika Stange ${ }^{3}$, Mareike Dietrich ${ }^{1}$, \\ Leonidas Apostolidis ${ }^{1}$, Angelika Freitag ${ }^{2}$, Claudia Trierweiler ${ }^{2}$, Carl von Gall ${ }^{4}$, Jennifer Ose ${ }^{5}$, Frederik Giesel ${ }^{6}$, Tim Frederik Weber ${ }^{7}$, \\ Florian Lordick ${ }^{8}$, Uwe Haberkorn ${ }^{6}$ and Dirk Jäger ${ }^{1}$
}

BACKGROUND: To assess the predictive value of early metabolic response ( $\triangle S U V)$ after short-term treatment with first-line cetuximab in patients (pts) with RAS-wt metastatic colorectal cancer (mCRC).

METHODS: In this prospective phase II study, RAS-wt mCRC pts received a single-agent cetuximab run-in therapy of 2 weeks. $\triangle$ SUV was assessed with FDG-PET/CT on days 0 and 14. Early clinical response (ECR) was evaluated with CT on day 56 after treatment with FOLFIRI-cetuximab. Primary endpoint was the predictive significance of $\triangle$ SUV for ECR. Secondary endpoints were PFS (progression free survival), OS and the influence of $\triangle$ SUV on survival.

RESULTS: Forty pts were enroled and 33 pts were evaluable for the primary endpoint. The CT response rate was $57.6 \%$. For responders, $\triangle$ SUV was significantly higher $(p=0.0092)$. A significant association of $\triangle$ SUV with ECR was found $(p=0.02)$. Median PFS was 11.7 months and median OS was 33.5 months with a 1 -year survival rate of $87.9 \%$. $\Delta$ SUV was found to significantly impact the hazard for OS $(p=0.045)$.

CONCLUSIONS: We demonstrate that cetuximab induces metabolic responses in mCRC pts. The study endpoint was met with the $\triangle S U V$ discriminating between responders and non-responders. However, these data should be validated in larger patient cohorts.

British Journal of Cancer (2018) 119:170-175; https://doi.org/10.1038/s41416-018-0152-4

\section{INTRODUCTION}

Currently, the median overall survival (OS) for patients with metastasised colorectal cancer (mCRC) is almost 2.5 years with response rates of $60 \%$ for the most active regimen. ${ }^{1}$ For the RASwildtype (wt) population, first-line anti-EGFR therapy is reported with improved response rates and increased resectability of metastases compared to anti-VEGF treatment, whereas the impact on survival is discussed controversially. ${ }^{2-4}$ However, as not all RASwt patients respond to anti-EGFR therapy, clinical exploration and validation of further biomarkers for this collective is warranted. ${ }^{5}$ Usually, treatment response evaluation in $\mathrm{mCRC}$ is based on morphological size criteria of tumour lesions as measured with computed tomography (CT) or magnetic resonance imaging (MRI), requiring a systemic treatment period of at least 6 to 8 weeks. Changes in the tumoural glucose metabolism are thought to precede morphological changes, and ${ }^{18} \mathrm{~F}$-fluorodeoxy-glucosepositron-emission-tomography/CT (FDG-PET/CT) has proven relevance in early predicting treatment response associated with improved survival in several solid tumour entities. ${ }^{6-8}$

We conducted a prospective phase II trial with sequential FDG$\mathrm{PET} / \mathrm{CT}$ before and after a 2-week run-in phase with single-agent cetuximab in RAS-wt first-line $m C R C$ patients. We aimed to evaluate the predictive significance of early changes in FDG uptake under single-agent cetuximab for the first morphological response as measured on day 56 under treatment with the FOLFIRI-cetuximab regimen.

\section{METHODS AND PATIENTS}

Trial design

This open-label, non-randomised phase II clinical trial was conducted at the National Center for Tumor Diseases (NCT), Heidelberg, Germany. The protocol was approved by the national regulatory authorities according to the requirements of $\S 40 \mathrm{ff}$.

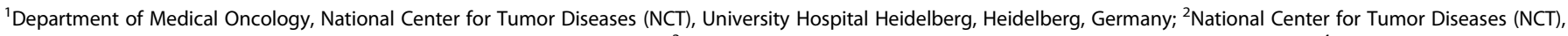

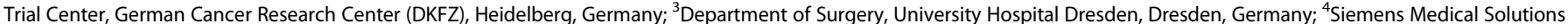

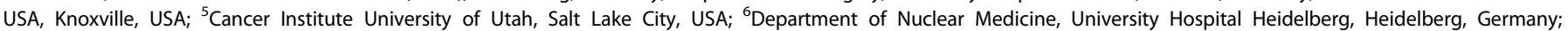

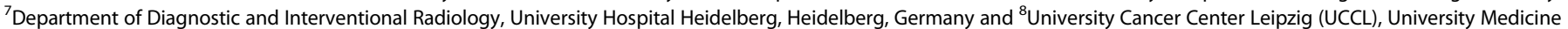
Leipzig, University of Leipzig, Leipzig, Germany

Correspondence: Anne Katrin. Berger (anne.berger@med.uni-heidelberg.de)

Received: 8 February 2018 Revised: 21 May 2018 Accepted: 29 May 2018

Published online: 2 July 2018 
AMG (German Drug Law). All aspects of the study were done in accordance with the declaration of Helsinki and the guidelines for Good Clinical Practice of the International Conference on Harmonization. An independent data monitoring committee monitored recruitment and safety.

\section{Patients}

Patients aged $\geq 18$ years with histologically confirmed $\mathrm{mCRC}$ were eligible for inclusion if they had no history of therapy with an EGFR targeting agent or chemotherapy for advanced disease, and measurable tumour lesions with a diameter no smaller than 1.0 $\mathrm{cm}$. An ECOG-performance status of 0 or 1 was mandatory as well as life expectancy $>12$ weeks and adequate haematological, renal and hepatic function. A KRAS-wt status (exon 2) of the tumours was requested. As in 2014, the approval for cetuximab was restricted to patients with both KRAS- (exons 2, 3,4) and NRAS(exons 2, 3, 4) wt status, the inclusion criteria of this trial were adapted accordingly. Full inclusion and exclusion criteria are given in Supplement 1.

\section{Study treatment}

Cetuximab was administered intravenously (i.v.) $400 \mathrm{mg} / \mathrm{m}^{2}$ on day 1 and $250 \mathrm{mg} / \mathrm{m}^{2}$ on day 8 as single-agent therapy within this study. For patients with any grade 3 or 4 toxicity (NCI-CTCAE), cetuximab could be withheld. For patients with severe skin reactions ( $\geq$ grade 3 ), cetuximab therapy could be withheld until the reaction had resolved to grade 2 . The duration of the study treatment was 2 weeks. Between day 14 and day 56, the patients were treated according to the FOLFIRI-cetuximab regimen as an active and approved first-line treatment for this patient cohort. According to the trial center's clinical standards, the FOLFIRIcetuximab regimen was applied every 2 weeks as follows: cetuximab $250 \mathrm{mg} / \mathrm{m}^{2}$ (days 1, 8), irinotecan $180 \mathrm{mg} / \mathrm{m}^{2}$ (day 1), folinic acid $400 \mathrm{mg} / \mathrm{m}^{2}$ (day 1), 5-fluorouracil $400 \mathrm{mg} / \mathrm{m}^{2}$ bolus (day 1) and $2400 \mathrm{mg} / \mathrm{m}^{2}$ for $46 \mathrm{~h}$ (day 1). Depending on the response on day 56, treatment was continued according to the choice of the responsible physician. It was recommended that, in case of response, treatment was continued with cetuximab and FOLFIRI until disease progression or patients were unable to tolerate the therapy.

\section{Imaging methodology and response analysis}

FDG-PET-CT imaging. At baseline (day 0) and at day 14, patients received an FDG-PET/CT for evaluation of early changes in tumour glucose uptake. Patients had to fast at least $6 \mathrm{~h}$ prior to the i.v. application of the radiopharmakon, and the blood glucose level should not exceed $150 \mathrm{mg} / \mathrm{dl}$. All patients received 20-40 mg butylscopolaminiumbromide i.v. to reduce bowl movements. A CT scan for attenuation correction was performed $1 \mathrm{~h}$ post tracer injection. Immediately after CT scanning, a wholebody PET was acquired in 3D (matrix: $164 \times 164$ ). For each bed position (16.2 cm, overlapping scale: $4.2 \mathrm{~cm}$ ) we used $4 \mathrm{~min}$. acquisition time with a $15.5 \mathrm{~cm}$ field of view (FOV). The emission data were corrected for randoms, scatter and decay. Reconstruction was conducted with an ordered subset expectation maximisation algorithm (OSEM) with 4 iterations/8 subsets and Gauss-filtered to a transaxial resolution of $4.2 \mathrm{~mm}$ at full width at half maximum (FWHM). Attenuation correction was performed using the low dose non-enhanced CT data. PET and CT were performed using the same protocol for every patient on a BIOGRAPH-6 PET/CT scanner (Siemens, Germany). For calculation of the standardised uptake value (SUV), circular regions of interest were drawn around areas with focally increased uptake in transaxial slices and automatically adapted to a threedimensional volume of interest at a $70 \%$ isocontour. At baseline, a diagnostic contrast-enhanced CT scan of the chest, abdomen and pelvis during the portal venous phase was included in the FDG-PET/CT protocol.
CT scanning and radiological assessment of response

At day 56 ( \pm 4 days), evaluation of morphological response was performed with a routine contrast-enhanced portal venous phase CT scan covering chest, abdomen and pelvis (Philips Brilliance iCT, The Netherlands). Response assessment was performed using the Response Evaluation Criteria in Solid Tumors version 1.1 (RECIST $1.1^{9}$ ). Early clinical response was defined as partial remission (PR) or complete remission (CR) according to RECIST 1.1.

Statistical design and sample size

The primary objective was the evaluation of the predictive significance of relative changes in SUV ( $\triangle$ SUV) in FDG-PET/CT during short-term single-agent treatment with cetuximab. Specifically, $\triangle$ SUV was defined as $100 \times\left(S_{U} V_{\text {baseline }}-S U V_{d 14}\right) / S U V_{\text {baseline }}$ All study patients were analysed for whom $\triangle S U V$ and response measurement were available. Secondary objectives included the duration of PFS and OS as well as the influence of $\triangle S U V$ and early clinical response on PFS and OS. PFS was defined as the time from study entry to objective tumour progression or death from any cause, whichever occurred first. PFS data were censored on the date of the last tumour assessment on study for patients who did not have primary disease progression (PD) and who did not die while on study. Patients lacking any tumour assessment after the first treatment administration had their PFS time censored on the date of first treatment administration with a duration of 1 day. OS was defined as the time from study entry to death from any cause. For living patients, time to death was censored at the time of last contact. The trial was designed to detect an AUC of $\triangle S U V=0.8$ with a power of $84.1 \%$ requiring $n=35$ evaluable patients. For detailed sample size calculation, see Supplement 2.

\section{Statistical methods}

The null hypothesis that $\Delta$ SUV has no predictive power for early clinical response was tested using the Wilcoxon rank-sum test (2sided, $a=5 \%$ ) comparing responders (CR, PR) with nonresponders. The degree of discrimination was quantified by means of the area under the ROC curve (AUC). The primary analysis was performed on all patients who were evaluable for the primary endpoint and who met the original inclusion criteria. The association of $\triangle S U V$ with the clinical response was analysed by logistic regression. Standard methods for survival analysis (KaplanMeier estimates of the survival curves, ${ }^{10}$ Cox proportional hazards regression ${ }^{11}$ ) were used in the analysis of time-to-event endpoints PFS and OS. The Wilcoxon rank-sum test was used to compare the $\triangle S U V$ values of clinical responders vs. clinical non-responders. The Wilcoxon signed-rank test was used to test changes in SUV from baseline to day 14 for statistical significance (2-sided, $a=5 \%$ ).

\section{RESULTS}

Recruitment and patient characteristics

From February 2011 to November 2014, 40 patients were enroled at our centre. All patients received the study medication and were analysed for safety (safety group). Four patients discontinued the trial (three patients due to adverse events (AEs), one patient withdrew consent). Two patients missed CT scan on day 56, and one patient did not show metabolic activity in FDG-PET/CT. Thus, 33 patients $(82.5 \%)$ were evaluable for the primary analysis (perprotocol-group). Information about the patient disposition is summarised in Fig. 1, which was prepared according to the current version of the CONSORT statement.

Patient population

Median age of patients was 62 years (range 32-78), and 15 patients were $\geq 65$ years. In the per-protocol-group, 16 patients (48.5\%) had distant metastases restricted to the liver. Two and three patients had metastases restricted to lung or lymph nodes, respectively. Twelve patients $(36.4 \%)$ had involvement of more 


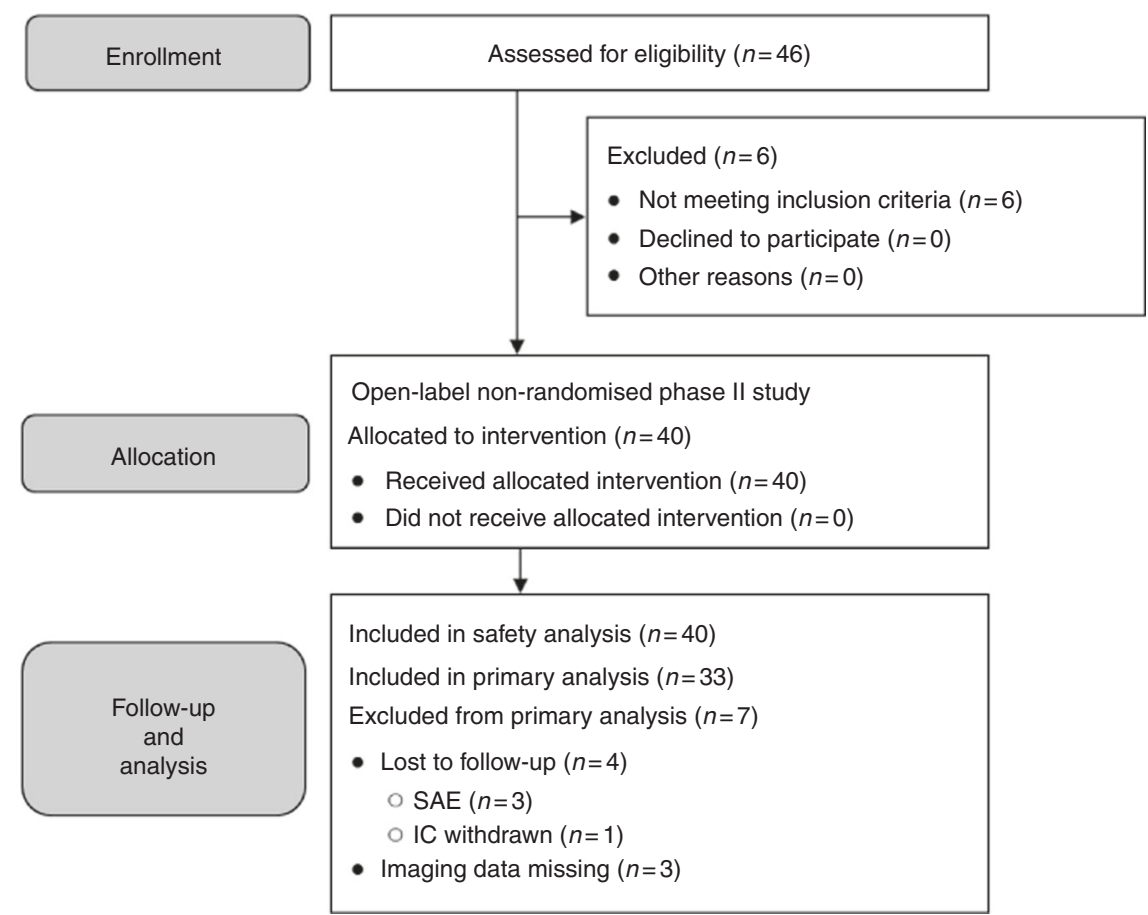

Fig. 1 Patient disposition: CONSORT flow diagram

than one organ site. Eleven patients (33.3\%) later underwent a resection approach for metastastic disease. Twenty-two patients $(66.7 \%)$ received second-line chemotherapy, and 11 patients (33.3\%) underwent three or more treatment lines. The first 26 patients were enroled under the original inclusion criteria (KRASwt exon 2), and 14 patients complied with full NRAS- and KRAS-wt status. For patients included prior to restriction of RAS criteria, an additional retrospective analysis for NRAS and full KRAS status was done whenever possible. For three patients of the per-protocolgroup, extended RAS data were missing (two patients without NRAS analysis and one patient with missing NRAS exon 4 analysis). In four patients, primarily unrecognised mutations in KRAS exons 3 or 4 or NRAS were found. Patients' baseline characteristics are given in Table 1. For detailed information on disease extent and resectability potential, see Supplement 3.

Safety

The rate of AEs under cetuximab treatment was well within expectations with the skin and the gastrointestinal tract being the most affected organ classes in $16(40 \%)$ and 26 patients (65\%), respectively. Fourteen patients experienced an AE of grade 3 or higher of which hypersensitivity reactions were the most frequent (three patients, 7.5\%). One patient was reported with an atypical pneumonia resulting in death without suspected relation to study treatment. AEs were collected and graded using the National Cancer Institute Common Terminology Criteria for Adverse Events, v 3.0.

Metabolic imaging and early morphological response CT scans on day 56 showed a PR in 17 patients $(51.5 \%)$ and a CR in two patients (6.1\%). Twelve patients (36.4\%; including all four patients with post-hoc identified RAS mutations) had a stable disease (SD) and two patients (6.1\%) had a PD. Thus, the early morphological response rate as defined in this trial $(\mathrm{PR}+\mathrm{CR})$ in the per-protocol-group was $57.6 \%$ under treatment with FOLFIRIcetuximab. With sequential FDG-PET/CT, the median SUVmax was 8.9 (range 3.0-19.8) at baseline and 6.0 (range 1.9-15.0) on day 14 . The median relative reduction in SUV ( $\triangle$ SUV) at day 14 for

\begin{tabular}{|ll|}
\hline Table 1. Patient characteristics & $N(\%)(n=40)$ \\
\hline Baseline characteristic & \\
\hline Age & $62(32-78)$ \\
$\quad$ Years (median, range) & \\
Sex & $31(77.5)$ \\
$\quad$ Male & $9(22.5)$ \\
Female & \\
Race & $38(95.0)$ \\
Caucasian/White & $2(5.0)$ \\
Oriental/Asian & \\
ECOG PS & $28(70.0)$ \\
0 & $12(30.0)$ \\
1 & \\
Primary Site & $20(50.0)$ \\
$\quad$ Rectum & $18(45.0)$ \\
Colon & $2(5.0)$ \\
$>1$ Primary site & \\
Site of metastases & $34(85.0)$ \\
Liver & $10(25.0)$ \\
Lung & $13(32.5)$ \\
Other & $66.5(0-2068)$ \\
Interval between first diagnosis and metastatic disease & \\
Days (median, range) & \\
&
\end{tabular}

all patients was $30.9 \%$ (range -33.3 to 65.7). For early morphological responders, the median $\triangle$ SUV was $42.3 \%$ (range -14.3 to 65.7 ) vs. a median $\Delta$ SUV of $20.3 \%$ (range -33.3 to 50.4 ) in the group of non-responders. The four non-wt patients had a median $\Delta$ SUV of $10.7 \%$ (range -8.9 to $20.9 \%$ ), see Supplementary Figure 1. 


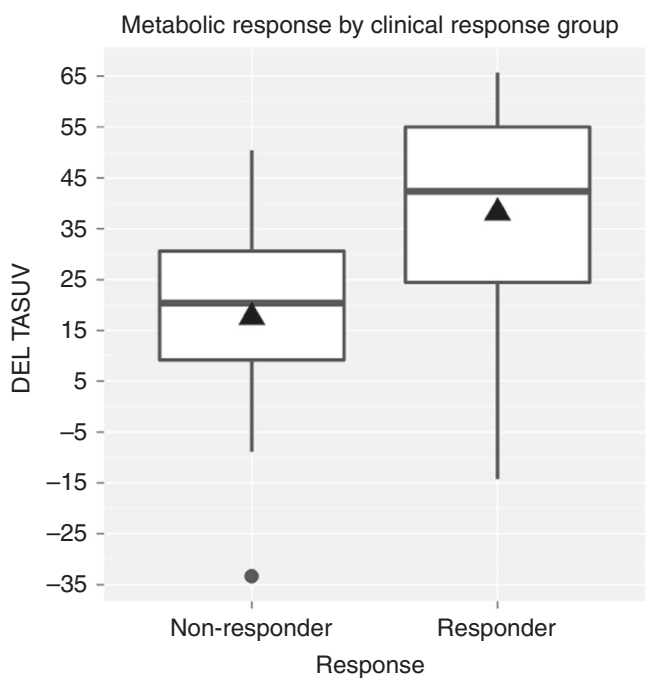

Fig. 2 Distribution of $\Delta$ SUV by response with group means (diamond), per-protocol-group

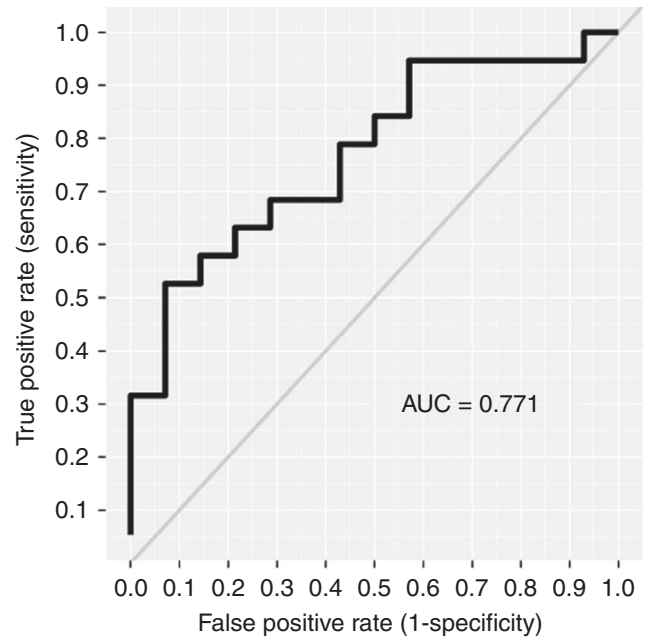

Fig. 3 ROC analysis for $\Delta S U V$ with respect to early clinical response, per-protocol-group

Proof-of-princle and effect size: the predictive power of $\Delta$ SUV The primary objective of this study was to evaluate the prognostic relevance of relative changes in SUV for early clinical response. The $\triangle S U V$ for responders was found to be significantly higher than for the group of non-responders, indicating that $\triangle S U V$ is-in principle-able to discriminate between non-responders and responders ( $p=0.0092$, Fig. 2 ). The degree of discrimination was quantified by means of the area under the ROC curve (AUC, Fig. 3). The AUC was determined to be 0.771 [95\% Cl: 0.609-0.933], indicating that the probability that a random responder has a higher value of $\triangle$ SUV than a random non-responder is $77.1 \%$. Logistic regression analysis indicated a statistically significant association of $\Delta$ SUV with early clinical response $(p=0.02$; OR $=$ 1.052 [95\% Cl: 1.007-1.098]). The adapted logistic model can be used to predict the clinical outcome based on the measured relative change in SUV (Fig. 4 d).

Patient follow-up and survival times

Patients were followed up for at least 24 months. The median duration of follow-up for the per-protocol-group was 42.7 months with 31 patients (93.9\%) experiencing disease progression. Two patients finished follow-up without event after 29.4 and 63.0 months, respectively. Median PFS was 11.7 months [95\% Cl:
7.3-14.8] (Fig. 4a) and the 1-year PFS rate was $45.5 \%$ [95\% Cl: 28.2-61.2]. Twenty patients (66.7\%) died during follow-up. Median OS was 33.5 months [95\% Cl: 21.1-39.8] (Fig. 4b) with a 1-year survival rate of $87.9 \%$ [95\%Cl: $70.9-95.3]$. $\Delta$ SUV was found to significantly impact the hazard for OS in univariable Cox regression analysis ( $p=0.045 ; \mathrm{HR}=0.98$ [95\%Cl: $0.95-0.999])$. In an explorative analysis, a $\triangle$ SUV $>20 \%$ (fitting with the EORTC recommendations for partial metabolic response ${ }^{12}$ ) was found to support the trend towards improved OS for metabolic responders (Fig. 4c).

\section{DISCUSSION}

Early treatment stratification for $\mathrm{mCRC}$ patients is relevant for both avoiding unnecessary toxicity and in terms of health care economics. Of note, modern antineoplastic treatments allow an increasing amount of conversion therapies in primarily irresectable mCRC cases, with an OS exceeding 5 years for the RAS-wt subgroup. ${ }^{4}$ A prolonged preoperative systemic treatment in these patients can raise the rate of surgical complications substantially, making a secondary curative approach impossible in the worst case scenario. ${ }^{13-15}$ Thus, oncologists are in need of reliable criteria for upfront identification of the most effective therapy for the patient and of tools that will allow for early response-adaptive treatment guidance. For radiologic assessment, early tumour shrinkage (ETS) was suggested as a potential predictive marker for long-term outcome in RAS-wt mCRC patients. ${ }^{16}$ Still, there are primary anti-EGFR non-responders in the RAS-wt collective, suggesting heterogeneity in EGFR signalling, and development of diagnostic tools predicting prognosis and therapy-response is of high interest. Innovative techniques such as liquid biopsies may provide a great potential for identification of further predictive molecular markers in this setting and may even allow for noninvasive monitoring of clonal dynamics. To date, prospective clinical validation is awaited, and recent guidelines do not recommend on molecular testing beyond extended RAS, BRAF and MSI-analysis. ${ }^{17}$ FDG-PET/CT is an imaging approach allowing metabolic response assessment with the SUV being the most widely applied semiquantitative parameter. $^{18}$ For response assessment, changes in the SUV ( $\triangle S U V)$ rather than the absolute SUV value have proven useful. ${ }^{19,} 20$ In $\mathrm{mCRC}$, only few prospective studies have evaluated the use of FDG-PET/CT for response monitoring. In older series on $\mathrm{mCRC}$ patients, metabolic response correlated with objective response but not with survival., ${ }^{81,22}$ So far, to our knowledge, no data on the effect of EGFR-blockade are available.

Our results show as a proof-of-principle that short-term singleagent treatment with cetuximab induces a high rate of metabolic tumour response in $\mathrm{mCRC}$ patients. This finding is in good accordance with data for other solid tumours under inhibition of the EGFR pathway. ${ }^{23},{ }^{24}$ Concerning RAS analysis, our results are consistent with those from previous studies showing that patients with mutant tumours are unlikely to benefit from anti-EGFR therapy. ${ }^{25}$

The $\triangle$ SUV at day 14 in our cohort differed significantly between early morphological responders (defined as PR or CR) and nonresponders. Thus, we could demonstrate that cetuximab induces (i) early relevant metabolic changes in $\mathrm{mCRC}$ that are (ii) predictive for later morphological response achieved by the combination of FOLFIRI plus cetuximab. Despite our rather small sample size, we found that $\triangle$ SUV had a significant impact on OS, suggesting that early metabolic response may hold prognostic value beyond prediction of early clinical response. The early FDG-PET/CT may prove useful as a tool for identification of $\mathrm{mCRC}$ patients benefitting from anti-EGFR treatment. It might serve as a complementary imaging strategy for rapid clinical verification of therapy-response, as needed for example in the neoadjuvant or conversion setting, even when an enlarged armamentarium of molecular signatures will 
a

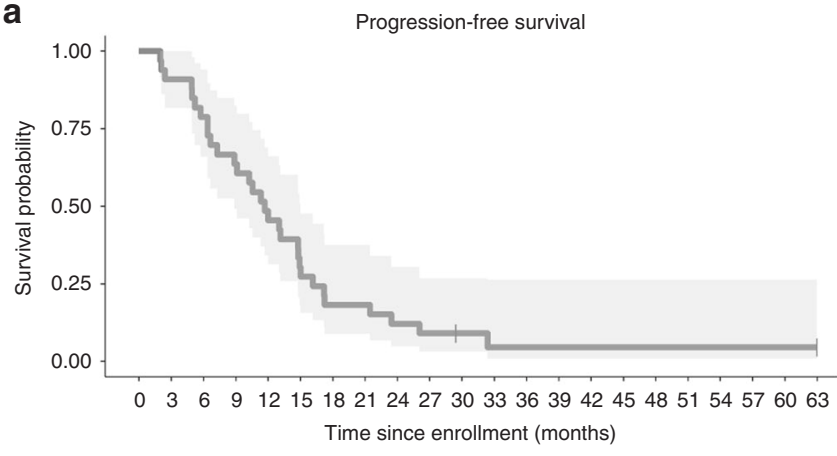

Number at risk

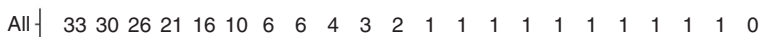

C

C Overall survival by metabolic response DELTASUV $=<20 \%-\geq 20 \%$

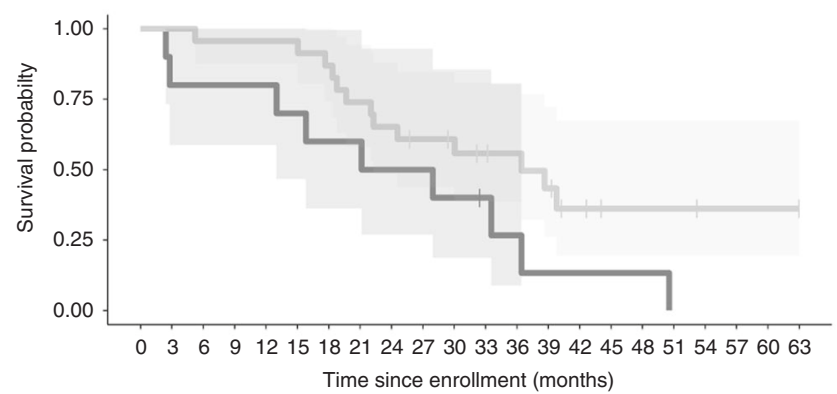

点

Number at risk

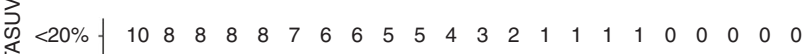

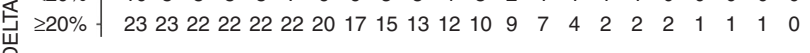

Fig. 4 a Kaplan-Meier estimates of PFS with number of subjects at risk, per-protocol-group. b Kaplan-Meier estimates of OS with number of subjects at risk, per-protocol-group. c Kaplan-Meier estimates of OS by reduction in SUV (\%), per-protocol-group. d Predicted probabilities of response for the logit model with 95\% confidence bounds, per-protocol-group

be available. In non-responders, an early change or escalation of the regimen (FOLFOX + Bevacizumab or FOLFOXIRI + Bevacizumab) might be beneficial in this setting. However, the question if a FDG-PET/CT-guided algorithm will translate into improved survival times or improved resection rates can only be answered by larger and randomised trials. The additional costs of FDG-PET/CT should be noted, but would probably be well outweighed by saving a very costly, toxic and quality-of-life-reducing therapy. However, it must be considered that FDG-PET/CT is not yet available in all regions and to all patients.

In addition, our results show that early FDG-PET/CT assessment is feasible for single-agent-targeted therapies in only a short-time treatment phase. Thus, it may prove useful considering early drug development with the provided information on immediate effects on tumour cells facilitating the identification of substances qualifying for further clinical evaluation.

However, our data of this small prospective patient collective can only be viewed as a pilot. The prospective definition of clinical meaningful cut-off points for $\triangle$ SUV allowing prediction of response and survival was beyond the scope of the REMOTUX trial, and the $\triangle$ SUV cut-off of $20 \%$ comes from an exploratory analysis. Further validation in independent patient cohorts is required to confirm our results and to answer the remaining questions.

\section{CONCLUSION}

Single-agent cetuximab treatment of only 14 days is able to induce metabolic responses in $\mathrm{MCRC}$ patients as measured by FDG-PET/CT. This early metabolic response is predictive for later morphologic response and may even predict survival times. These

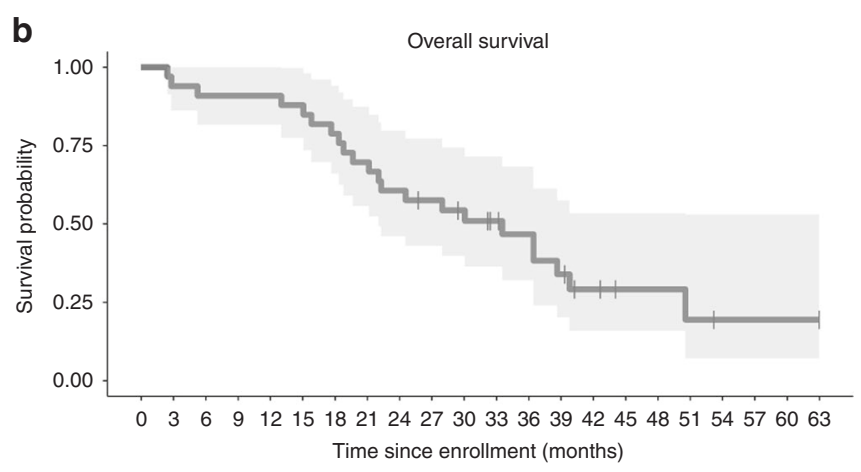

Number at risk

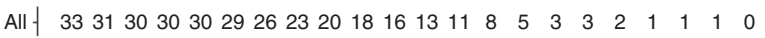

d

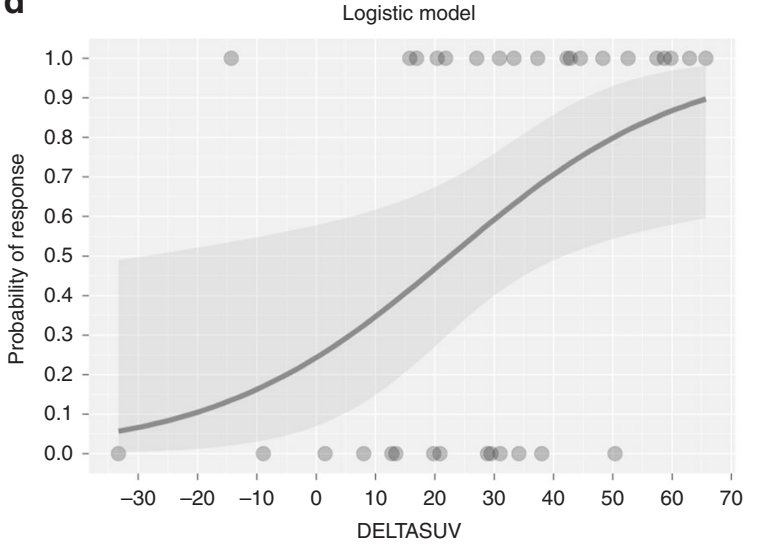

results could impact on future treatment stratification in RAS-wt mCRC patients with implementation of early FDG-PET/CT as an additional and helpful tool for optimal treatment guidance, especially in the conversion setting. However, futher prospective trials are needed to definitely answer the question of an FDG-PET/ CT-guided treatment algorithm in $\mathrm{mCRC}$ and to date, its use cannot be recommended in a routine setting.

Registration and protocol participate

The study was registered under EUDRA-CT: 2009-013279-23 and ISRCTN 75334801, and the protocol was published previously. $^{26}$

\section{AUTHOR CONTRIBUTIONS}

Conception and design: F.L., A.K.B., U.A., C.G., J.O., A.S. Acquisition of data: A.K.B., G.M.H., C.G., M.D., L.A., T.F.W., F.G. Analysis and interpretation of data: A.K.B., S.L., G.M.H., F.L., F.G., DJ. Writing, review, and/or revision of the manuscript: A.K.B., S.L., G.M.H., C.G., A.S., T.F. W., F.L., U.H., D.J. Administrative, technical, or material support: S.L., U.A., A.F., C.T.

\section{ADDITIONAL INFORMATION}

Supplementary information is available for this paper at https://doi.org/10.1038/ s41416-018-0152-4.

Competing interests: The authors declare no competing interests.

Ethics approval: The study was approved by the local Ethics Committee (Ethics Committee University of Heidelberg). The protocol was approved by the national regulatory authorities according to the requirements of §40ff. AMG (German Drug Law). 
All aspects of the study were done in accordance with the declaration of Helsinki and the guidelines for Good Clinical Practice of the International Conference on Harmonization.

Availability of data and materials: All data are available via the corresponding author and the NCT trial centre.

Funding: This study is an investigator-initiated trial, funded by Merck Pharma GmbH The contract between the financial sponsor and the University Hospital of Heidelberg leaves the full responsibility for the scientific work, the management of data, and for analysis and publication to the investigators.

Note: This work is published under the standard license to publish agreement. After 12 months the work will become freely available and the license terms will switch to a Creative Commons Attribution 4.0 International (CC BY 4.0).

\section{REFERENCES}

1. Cremolini, C. et al. FOLFOXIRI plus bevacizumab versus FOLFIRI plus bevacizumab as first-line treatment of patients with metastatic colorectal cancer: updated overall survival and molecular subgroup analyses of the open-label, phase 3 TRIBE study. Lancet Oncol. 16, 1306-1315 (2015).

2. Heinemann, V. et al. FOLFIRI plus cetuximab versus FOLFIRI plus bevacizumab as first-line treatment for patients with metastatic colorectal cancer (FIRE-3): a randomised, open-label, phase 3 trial. Lancet Oncol. 15, 1065-1075 (2014).

3. Schwartzberg, L. S. et al. PEAK: a randomized, multicenter phase II study of panitumumab plus modified fluorouracil, leucovorin, and oxaliplatin (mFOLFOX6) or bevacizumab plus mFOLFOX6 in patients with previously untreated, unresectable, wild-type KRAS exon 2 metastatic colorectal cancer. J. Clin. Oncol. 32, 2240-2247 (2014).

4. Venook, A. P. et al. CALGB/SWOG 80405: Phase III trial of irinotecan/5-FU/leucovorin (FOLFIRI) or oxaliplatin/5-FU/leucovorin (mFOLFOX6) with bevacizumab (BV) or cetuximab (CET) for patients (pts) with KRAS wild-type (wt) untreated metastatic adenocarcinoma of the colon or rectum (MCRC). J. Clin. Oncol. 32, LBA3-LBA3 (2014).

5. Fakih, M. G. Metastatic colorectal cancer: current state and future directions. J. Clin. Oncol. 33, 1809-1824 (2015).

6. Lordick, F. et al. PET to assess early metabolic response and to guide treatment of adenocarcinoma of the oesophagogastric junction: the MUNICON phase II trial. Lancet Oncol. 8, 797-805 (2007).

7. Grootjans, W. et al. PET in the management of locally advanced and metastatic NSCLC. Nat. Rev. Clin. Oncol. 12, 395-407 (2015).

8. Hendlisz, A. et al. Serial FDG-PET/CT for early outcome prediction in patients with metastatic colorectal cancer undergoing chemotherapy. Ann. Oncol. 23, 1687-1693 (2012).

9. Eisenhauer, E. A. et al. New response evaluation criteria in solid tumours: revised RECIST guideline (version 1.1). Eur. J. Cancer 45, 228-247 (2009).

10. Kaplan, E. L. \& Meier, P. Nonparametric estimation from incomplete observations. J. Am. Stat. Assoc. 53, 457-481 (1958).

11. Cox, D. R. Regression models and life-tables. J. R. Stat. Soc. Series B (Methodol.). 34, 187-220 (1972).
12. Young, $\mathrm{H}$. et al. Measurement of clinical and subclinical tumour response using [18F]-fluorodeoxyglucose and positron emission tomography: review and 1999 EORTC recommendations. European Organization for Research and Treatment of Cancer (EORTC) PET Study Group. Eur. J. Cancer 35, 1773-1782 (1999).

13. Kishi, Y. et al. Extended preoperative chemotherapy does not improve pathologic response and increases postoperative liver insufficiency after hepatic resection for colorectal liver metastases. Ann. Surg. Oncol. 17, 2870-2876 (2010).

14. Vauthey, J. N. et al. Chemotherapy regimen predicts steatohepatitis and an increase in 90-day mortality after surgery for hepatic colorectal metastases. J. Clin. Oncol. 24, 2065-2072 (2006).

15. Hubert, C. et al. Nodular regenerative hyperplasia: a deleterious consequence of chemotherapy for colorectal liver metastases? Liver. Int. 27, 938-943 (2007).

16. Piessevaux, H. et al. Use of early tumor shrinkage to predict long-term outcome in metastatic colorectal cancer treated with cetuximab. J. Clin. Oncol. 31, 3764-3775 (2013).

17. Sepulveda, A. R. et al. Molecular biomarkers for the evaluation of colorectal cancer: guideline from the American Society for Clinical Pathology, College of American Pathologists, Association for Molecular Pathology, and the American Society of Clinical Oncology. J. Clin. Oncol. 35, 1453-1486 (2017).

18. Maffione, A. M. et al. Are PREDIST criteria better than PERCIST criteria as a PET predictor of preoperative treatment response in rectal cancer? Nucl. Med. Commun. 35, 890-892 (2014).

19. Huh, J. W., Min, J. J., Lee, J. H., Kim, H. R. \& Kim, Y. J. The predictive role of sequential FDG-PET/CT in response of locally advanced rectal cancer to neoadjuvant chemoradiation. Am. J. Clin. Oncol. 35, 340-344 (2012).

20. Maffione, A. M. et al. Early prediction of response by (1)(8)F-FDG PET/CT during preoperative therapy in locally advanced rectal cancer: a systematic review. Eur. J. Surg. Oncol. 40, 1186-1194 (2014).

21. Bystrom, P. et al. Early prediction of response to first-line chemotherapy by sequential [18F]-2-fluoro-2-deoxy-D-glucose positron emission tomography in patients with advanced colorectal cancer. Ann. Oncol. 20, 1057-1061 (2009).

22. Skougaard, K., Nielsen, D., Jensen, B. V., Pfeiffer, P. \& Hendel, H. W. Early (18)FFDG-PET/CT as a predictive marker for treatment response and survival in patients with metastatic colorectal cancer treated with irinotecan and cetuximab. Acta Oncol. 55, 1175-1182 (2016).

23. Schmitz, S. et al. Tumour response and safety of cetuximab in a window preoperative study in patients with squamous cell carcinoma of the head and neck. Ann. Oncol. 24, 2261-2266 (2013).

24. Sunaga, N. et al. Usefulness of FDG-PET for early prediction of the response to gefitinib in non-small cell lung cancer. Lung Cancer 59, 203-210 (2008).

25. Hecht, J. R. et al. Extended RAS analysis for anti-epidermal growth factor therapy in patients with metastatic colorectal cancer. Cancer Treat. Rev. 41, 653-659 (2015).

26. Berger, A. K. et al. A phase II study for metabolic in vivo response monitoring with sequential 18FDG-PET-CT during treatment with the EGFR-monoclonal-antibody cetuximab in metastatic colorectal cancer: the Heidelberg REMOTUX trial. BMC Cancer 12, 108 (2012). 